\title{
RESPONSABILIDAD Y REPUTACIÓN SOCIAL CORPORATIVAS: COINCIDENCIAS $Y$ DIFE- RENCIAS ENTRE LOS INDICADORES DE SU EVALUACIÓN
}

\author{
MIGUEL ÁNGEL NICOLÁS OJEDA \\ Universidad Católica San Antonio de Murcia \\ Murcia, Espanha \\ E-mail:manicolas@ucam.edu \\ DAVID SÁNCHEZ HERVÁS \\ Universidad Católica San Antonio de Murcia \\ Murcia, Espanha \\ E-mail: dsanchez@ucam.edu
}


RESPONSABILIDAD Y REPUTACIÓN SOCIAL CORPORATIVAS: COINCIDENCIAS Y DIFERENCIAS ENTRE LOS INDICADORES DE SU EVALUACIÓN

Resumen: Este trabajo es un análisis comparado entre los indicadores que evalúan la responsabilidad social corporativa y los indicadores de evaluación de la reputación. Los referentes para el análisis son la guía GRI y el monitor de reputación corporativa español Merco. Los resultados del trabajo muestran los criterios de evaluación coincidentes y una discusión acerca de los objetivos y funciones que motivan la realización de las memorias de responsabilidad social encaminadas a ser más un fin al servicio de la reputación que un instrumento de transparencia y sostenibilidad.

Palabras clave: Reputación Social Corporativa, Responsabilidad Social Corporativa, Indicadores de evaluación, GRI, Merco.

\section{A RESPONSABILIDADE SOCIAL E REPUTAÇÃO CORPORATIVA: SEMELHANÇAS E DIFERENÇAS ENTRE OS INDICADORES DE AVALIAÇÃO}

Resumo: Esta análise compara indicadores de responsabilidade social corporativa (GRI) e os indicadores de avaliação de reputação (monitor Merco). Os resultados do trabalho refletem a sobreposição entre os critérios. Finalmente, os autores discutem os objetivos e funções das memórias de responsabilidade social. Eles são uma ferramenta para o gerenciamento de reputação social ou uma ferramenta de transparência e sustentabilidade das organizações?

Palavras chave: Reputação, Responsabilidade Social Corporativa, os indicadores de avaliação, GRI, Merco.

SOCIAL CORPORATE RESPONSIBILITY AND CORPORATE REPUTATION: SIMILARITIES AND DIFFERENCES BETWEEN INDICATORS OF EVALUATION

Abstract: This analysis compares indicators of corporate social responsibility (GRI) and the evaluation indicators of reputation (Merco monitor). Work results reflect the overlap between criteria. Finally, the authors discuss the objectives and functions of the memories of social responsibility, they are a tool for managing social reputation or a tool for transparency and sustainability of organizations?

Keywords: Corporate Reputation, Corporate Social Responsibility, Evaluation indicators, GRI, Merco. 


\section{INTRODUCCIÓN}

Como nos recuerdan Carreras, Alloza y Carreras (2013, p.27), la gestión de la reputación corporativa ha incrementado su importancia entre las organizaciones en los últimos años, así, para los autores el actual ciclo económico debería denominarse "economía de los intangibles y de la reputación corporativa". Cada día son más las organizaciones que muestran interés por esta forma de ser y de gestionar, ya que en el siglo XXI, el valor inmaterial de las organizaciones gira en torno a las marcas, imagen, conocimiento, habilidades de la gente, la responsabilidad social corporativa y la reputación corporativa (CARRILLO, CASTILLO y TATO, 2008).

Una breve revisión de las definiciones del concepto de reputación corporativa muestra que no hay una única definición ni cohesión entre los elementos que la definen. Para Orozco y Ferré (2012, p.6) “los teóricos más relevantes que han tratado el tema no han llegado a un consenso sobre su definición" y para Martínez y Olmedo (2009) esta falta de consenso general es debida a que se aborda y define desde distintas disciplinas. Fombrun y Van Riel (1997) afirman que la reputación se desarrolla desde el nivel interno de la institución, a partir de la actuación y la historia de la organización, y se conforma a través de la percepción que los stakeholders tienen de ella. Por otro lado, Villafañe (2004) añade un matiz a esta definición, la necesidad de gestionarla y comunicarla para que genere valor. Para Martín, Fernández y Alameda (2010) las consecuencias de la generación de valor es, además de obtener una mejor relación con los stakeholders, la percepción de notables beneficios económicos. Concluimos que la reputación es la percepción (valoración) que los stakeholders tienen de una organización a partir su comportamiento, de gestión pasada, presente y futura y de la gestión de su comunicación para favorecer una percepción positiva de su imagen. Para Carreras, Alloza y Carreras (2013) la reputación permite identificar las diferencias entre la realidad de la organización, la realidad comunicada y la realidad percibida y valorada por los grupos de interés.

Existen mecanismos capaces de evaluar la gestión la reputación se puede identificar, cuantificar e incluso modificar "la reputación corporativa no solo se puede medir y es mesurable, sino que se puede verificar" (VILLAFAÑE, 2005, p.32). "La gestión de la reputación establece una dinámica mediante la cual la organización se dota de herramientas de investigación y de escucha para conocer las percepciones, opiniones y las evaluaciones que los grupos 
de interés hacen de ella" (CARRERAS, ALLOZA y CARRERAS, 2013, p.38). Sin embargo, existe dificultad para medir y gestionar la reputación debido a su "escaso consenso en cuanto a conceptualización y medición", (MARTíNEZ y OLMEDO, 2009, p.139). Uno de los componentes con mayor influencia en la conformación de la reputación es la responsabilidad social corporativa, como afirman Martín, Fernández y Alameda (2010, p.7) “en el ámbito de la reputación corporativa, la responsabilidad social de la empresa se articula como una de sus dimensiones fundamentales". Bowen (1953), considerado el padre de la responsabilidad social, sienta las bases de este concepto. Para este autor los empresarios deben adquirir nuevas responsabilidades más allá de los beneficios económicos, impulsando políticas corporativas y siguiendo líneas de acción beneficiosas para la sociedad. En esta línea Martín, Fernández y Alameda (2010) indican que la responsabilidad social no son meras acciones aisladas y con objetivos filantrópicos, sino que debe estar integrada en las políticas de la organización con una visión a largo plazo, por sus posibles efectos sobre el rendimiento de la empresa. Estos autores no sólo sostienen el planteamiento aportado por Bowen sino que además afirman que "la responsabilidad social es una forma de asumir la dirección estratégica de la empresa" (MARTíN, FERNÁNDEZ y ALAMEDA, 2010, p.6). El hecho de que la responsabilidad social esté integrada en las políticas de la organización, tenga una visión a largo plazo y sea una forma de asumir la dirección estratégica de la empresa lo relaciona estrechamente con la reputación, convirtiéndose en una variable imprescindible, pero no la única. Así Villafañe (2009) asegura que, teniendo en cuenta los datos suministrados por los estudios realizados por los monitores internacionales dedicados a valorar la reputación corporativa de las instituciones, ésta se configura a partir de otros indicadores que tienen más peso que la gestión de la responsabilidad corporativa de la empresa, como es por ejemplo la calidad de la oferta comercial.

En este punto surge la reflexión acerca de la transparencia y los objetivos que determinan la gestión de la responsabilidad social corporativa. Fernández (2007, p.45) considera que Europa ha copiado el modelo estadounidense sobre la gestión de la responsabilidad social

en Estados Unidos gran parte de las decisiones de los altos dirigentes empresariales a favor de la responsabilidad social de sus compañías parecen consistir en actuaciones instrumentales al servicio final del valor accionarial, de acuerdo con el modo financiero ilustrado y la también de moda <<filantropía estratégica >>. 
En este marco, el informe o memoria de responsabilidad social corporativa se ha convertido en una de las principales acciones para comprobar la política que mantiene la institución en relación a sus programas de responsabilidad. En las principales conclusiones expuesta en el estudio "Los informes de responsabilidad: presente y futuro" (2013) se resalta que el interés más notable que mueve a los directivos de las empresas a realizar el informe de sostenibilidad es contribuir a mejorar la imagen y la reputación corporativa y no tanto servir como herramienta de transparencia y rendición de cuentas de las medidas adoptadas por las empresa para integrar plenamente la responsabilidad social de la estructura de gestión. También se afirma, por parte de los expertos del estudio, que la mejora de la imagen y la reputación así como su función como herramienta de comunicación, son las principales razones que llevan a las empresas a realizar informes de sostenibilidad.

Desde esta perspectiva la finalidad por la que se informa, a través de los informes de sostenibilidad, de las acciones emprendidas por la empresa, debiera ser la del compromiso de las empresas hacia lo social y la transparencia informativa hacia sus stakeholders, sin embargo no parece esta ser su verdadera motivación. En este mismo sentido Moneva (2005) afirma que el consenso entre las diferentes definiciones del término responsabilidad ya no solo reside en el desarrollo económico, ya que ahora la protección del medio ambiente y el bienestar social deben ser objetivos a tener en cuenta por las organizaciones.

\section{DESARROLLO}

\subsection{Objeto de estudio y metodología}

El principal propósito de este trabajo es analizar y comparar aquellos puntos de coincidencia y diferencia entre los instrumentos utilizados para informar sobre los programas de responsabilidad social corporativa y los instrumentos utilizados para medir la reputación social corporativa o empresarial

Para tal, se desarrolla un análisis descriptivo y comparado de las estructuras y contenidos que incorporar los informes de responsabilidad. Hemos utilizado como referencia la guía GRI.

Para el análisis de los indicadores que miden la reputación corporativa, hemos analizado de manera general los parámetros de los monitores World's Most Admired Companies y Global RepTrak Pulse y de manera detallada el monitor de reputación social corporativa español Merco. 


\subsection{Análisis}

Cómo nos recuerda Dopazo (2012, p.294), el informe de responsabilidad social corporativa es aquel documento en el que las empresas explican los principios, las políticas y los objetivos que desempeñan en materia económica, ambiental y social, "supone, por tanto, un destacado ejercicio de transparencia, permite la autoevaluación y además la evaluación por parte de terceros". La autora destaca claramente que su principal función de este informe es la transparencia a modo de rendimiento de cuentas en materia de responsabilidad hacia sus grupos de interés, pero también reconoce el valioso interés que este documento supone como herramienta de comunicación para contribuir a la gestión de la reputación corporativa.

Así, la Responsabilidad Social se comprende, al menos, desde una doble dimensión: A. Políticas internas y externas, normativas locales e internaciones y principios organizacionales que dictaminen el quehacer responsable de la empresa. B. Normativas, guías y directrices locales e internacionales que determinen la comunicación del quehacer responsable de la empresa, es decir, directrices que encomienden los contenidos y la forma que debe adoptar el informe de responsabilidad social corporativa, primero como herramienta de transparencia y segundo como herramienta de apoyo a la gestión de la reputación corporativa.

Berbel, Reyes y Gómez (2007) recogen una clasificación en cuatro niveles de iniciativas internacionales para gestionar la responsabilidad social de las organizaciones: Nivel 1: Iniciativas que dictaminan las guías axiológicas y ontológicas de la RSO. Compromisos adquiridos por los países en el seno de la ONU, la Declaración Universal de Derechos Humanos, la Convención de los Derechos del niño, Organización para la Cooperación y el Derecho Económico, etc. Nivel 2: Desarrollo de la comunicación e interacción con los grupos de interés. Se recogen todos los documentos con las pautas para la elaboración de los informes de responsabilidad. Nivel 3. Directrices para modificar la acción y la gestión de las organizaciones y favorecer la praxis de la responsabilidad. Nivel 4. Iniciativas para evaluar las acciones de responsabilidad emprendidas. Incluye las iniciativas dispuestas para promover el conocimiento de la epistemología y las acciones de responsabilidad desarrolladas.

La elaboración del informe de responsabilidad social se ajusta al tercer nivel expuesto por Berbel, Reyes y Gómez. En este sentido, la guía de Global Reporting Initiative (GRI) se ha convertido en el modelo más reconocido. 
Aún así, son diversas las recomendaciones, guías y normativas que deben tener en cuenta los responsables de su elaboración. Como reflejo del Observatorio de Responsabilidad Social Corporativa (www.observatoriorsc.org) recomienda tener en cuenta: Declaración Universal de los Derechos Humanos. Normas de Naciones Unidas sobre Responsabilidades de las Empresas Transnacionales y otras Empresas Comerciales en la esfera de los Derechos Humanos. Líneas Directrices de la OCDE para Empresas Multinacionales. Informe del Foro de Expertos en Responsabilidad Social de las Empresas. Informe de la Subcomisión para potenciar y promover la Responsabilidad Social de las Empresas. Report on CSR: a new partnership. Normas sobre las responsabilidades de las empresas transnacionales y otras empresas comerciales en la esfera de los derechos humanos. Consejo Económico y Social de Naciones Unidas. Líneas Directrices de la OCDE para Empresas Multinacionales. Organisation for Economic Co-operation and Development.

GRI se define como entidad líder en promover el uso de las memorias sobre responsabilidad para fomentar que las organizaciones sean más sostenibles y contribuyan a un desarrollo sostenible. En su web describen los principios que una organización debe seguir para elaborar sus memorias de sostenibilidad. En ellos se delimita que los propósitos de las memorias son: Valorar el desempeño de una organización en materia de sostenibilidad en relación a las leyes, normas o códigos que regulen o afecten a su gestión o tarea. También para valorar la influencia de una organización en el desarrollo sostenible y comparar esta y su desempeño con el de otras organizaciones.

Para GRI toda memoria de responsabilidad debe sustentarse en: 1. Principios para definir los contenidos, 2. Principios para verificar la calidad de la memoria. 3. Orientaciones sobre la cobertura de la memoria. Estos principios, a su vez de subdividen y determinan el sentido de los indicadores de evaluación. De forma resumida, exponemos los apartados, subapartados e indicadores de la guía para establecer una comparativa con los indicadores de los sistemas de evaluación de la reputación corporativa:

a. Estrategia y análisis: Aspectos relacionados con las decisiones empresariales, los impactos, riesgo y oportunidades de la organización en materia de sostenibilidad y su repercusión sobre los stakeholders y la economía de la organización.

b. Perfil de la organización: Nombre, marcas, productos y/o servicios, estructura, localización, número y nombre de países en los que opera, naturaleza y forma jurídica, mercados servidos, desglose geográfico, sectores y 
clientes, dimensión, cambios significativos, premios y distinciones.

c. Parámetros de la memoria: miden los contenidos y su modo de presentación en la memoria: Periodo evaluado, fecha de la memoria anterior., ciclo de presentación de memoria, contenidos, materialidad, aspectos incluidos, grupos de interés, cobertura (países, divisiones, filiales), limitaciones de alcance o cobertura, información sobre negocios conjuntos, información sobre la recopilación de los indicadores, cambios (de expresión y motivos, de cobertura y métodos), tabla de contenidos, explicación del proceso de verificación de la memoria.

d. Gobierno, compromisos y participación de los grupos de interés: Estructura, funciones complementarias del presidente, número y sexo de los miembros del máximo órgano de gobierno, métodos para comunicar recomendaciones de los empleados y accionistas a los órganos de gobierno, vínculo entre la retribución de los miembros del órgano de gobierno y el desempeño de la organización, procedimientos para evitar conflictos, procedimiento para determinar la composición del órgano de gobierno, desarrollo interno de la misión y valores, procedimientos para supervisar el desempeño económico, ambiental y social, procedimientos para evaluar el desempeño económico, ambiental y social, descripción de los planteamientos de precaución, principios o programas sociales, ambientales y económicos desarrollados, asociaciones a las que pertenece o apoya la organización, relación de grupos de interés, bases para su identificación y selección, criterios para su inclusión, preocupaciones y aspectos de interés tras su participación.

e. Enfoque de gestión e indicadores de desempeño y descripción: Indicadores de desempeño de las dimensiones económica, medioambiental y social (se divide en: laborales, derechos humanos, sociedad y responsabilidad sobre productos).

e.1 Indicadores para medir la dimensión Económica: Valor económico directo. Consecuencias financieras y riesgos derivados del cambio climático. Cobertura de las obligaciones derivadas del programa de beneficios sociales. Ayudas financieras recibidas de gobiernos. Rango de salarios (por sexos y comparación con el salario mínimo local). Gastos en proveedores locales. Procedimientos para la contratación local y proporción de directivos de origen local. Inversiones y servicios prestados en beneficio público. Impactos económicos indirectos y su alcance.

e.2 Dimensión ambiental: Peso y volumen de los materiales utilizados y porcentaje de que son valorizados. Consumo directo e indirecto de energía. 
Ahorro general debido a las mejoras de eficiencia. Iniciativas para proporcionar productos eficientes. Iniciativas para reducir el consumo indirecto de energía. Captación total de agua por fuentes y número han sido afectas por su captación. Volumen y \% de agua que ha sido reciclada y reutilizada. Descripción de terrenos cercanos o ubicados dentro de espacios protegidos. Impactos en los espacios naturales derivados de la actividad de la organización. Habitas protegidos o restaurados. Estrategias y acciones implantadas y planificadas para la gestión del impacto en la biodiversidad. Número de especies protegidas ubicadas en las áreas afectadas por las acciones de la organización. Emisiones totales de gases de efecto invernadero. Otras emisiones indirectas de efecto invernadero. Iniciativas para reducir las emisiones de gases de efecto invernadero y las reducciones logradas. Emisiones de sustancias destructoras de la capa de ozono. NO y SO significativas. Volumen total de aguas residuales vertidas. Peso total de residuos gestionados. Número total y volumen de derrames accidentales. Peso de los residuos gestionados. Número y tamaño de hábitats afectados por vertidos de agua de la organización. Iniciativas para mitigar los impactos ambientales de productos y servicios. Porcentaje de productos vendidos y materiales de embalaje recuperados al final de su vida. Coste de las multas y sanciones por incumplimiento de normas medioambientales. Impactos medioambientales derivados del trasportes de productos y otros bienes utilizados para las actividades. Desglose por tipo de gasto e inversiones medioambientales.

e.3. Dimensión Social.

e.3.1. Indicadores del desempeño de prácticas laborales y ética del trabajo: Desglose de trabajadores por tipo de empleo, contrato, región y sexo. Número total de empleados, nuevas contrataciones y rotación. Beneficios sociales para empleados. Porcentaje de empleados cubiertos por un convenio colectivo. Periodo mínimo de preaviso relativos a cambios organizativos. Porcentaje del total de trabajadores que están representados en comités de salud y seguridad. Tasas de absentismo, enfermedades, profesionales, victimas mortales. Programa de educación, formación, asesoramiento, prevención y control de riesgo aplicados a los trabajadores. Acuerdos de salud y seguridad en acuerdos formales con sindicatos. Promedio de horas de formación. Programas de gestión de habilidades y de formación. Porcentaje de empleados que reciben evaluaciones regulares del desempeño y de desarrollo profesional por sexo. Composición de los órganos de gobierno y plantilla. Relación entre salario base de los hombres comparado con el de 
las mujeres y categoría. Niveles de reincorporación al trabajo y de retención tras bajas por maternidad o paternidad.

e.3.2. Indicadores del desempeño de los Derechos humanos: Contratos (número y \%) que incluyan cláusulas que incorporen preocupación en materia de derechos humanos. \% de proveedores, contratistas y otros socios investigados en materia de derechos humanos. Horas de formación y \% del personal de seguridad formado sobre políticas y procedimientos relacionados con los derechos humanos. Incidentes de discriminación y medidas adoptadas. Operaciones y proveedores significativos que corren riesgo de romper los derechos a la libertad de asociación y medidas adoptadas. Operaciones y proveedores relacionados con la explotación infantil, con operaciones de trabajo forzoso y medidas adoptadas. Incidentes relacionados con violaciones de los derechos de los indígenas y medidas adoptadas Operaciones (\% y número) que han sido evaluadas en materia de derechos humanos. Quejas relacionadas con los derechos humanos, tratadas y resueltas.

e.3.3. Indicadores del desempeño de Sociedad: Operaciones de desarrollo y evaluación del impacto en las que participa la comunidad local. Operaciones con impactos negativos en las comunidades locales. Medidas de respuesta a incidentes de corrupción. Posición en políticas públicas y en actividades de "lobbying". Aportaciones financieras a partidos políticos o a instituciones relacionadas. Número de prácticas monopolísticas. Valor monetario de sanciones por incumplimiento de la ley.

e.3.4. Indicadores del desempeño de Responsabilidad de productos: Ciclo de vida del producto y servicios e impactos en la salud y seguridad de los clientes. Porcentaje de categorías evaluadas. Incidentes por el incumplimiento de la y los códigos de regulación de los productos en salud y seguridad. Información sobre productos y servicios requerida por los procedimientos en vigor. Incumplimientos (normativas, ley, códigos) de información y etiquetado. Acciones de satisfacción del cliente. Acciones de cumplimiento de las leyes (normativas, códigos) mencionadas en comunicaciones de marketing. Incidentes por el incumplimiento de las normativas de comunicación de marketing. Reclamaciones por privacidad y fuga de datos personales. Coste de multas significativas por incumplimiento de la norma de suministro y uso de productos y servicios.

En la segunda fase del estudio analizamos los índices utilizados por los organismos más acreditados dedicados a evaluar la reputación de las organizaciones y elaborar los rankings de reputación. Según Orozco y Ferré 
(2012, p.8) éstos sistemas "han ido creciendo por la necesidad que tienen los empresarios de determinar numéricamente los efectos de la implementación de las estrategias de comunicación y diálogo con sus stakeholders". Sin embargo, existen voces críticas respecto al verdadero valor de estos índices ya que cuentan con unas métricas insuficientes "es importante señalar la dificultad de realizar un índice de medida de la reputación adaptado a cualquier sector o actividad", (MARTíNEZ Y OLMEDO, 2009, p.140). Son diversos los ejemplos de organizaciones que, tras obtener buenas calificaciones en los ranking de prestigiosos monitores de reputación, han demostrado ser auténticos desastres en su gestión (Lehman Brothers / Bankia), "sobre todo por sus muy marcadas deficiencias en materia de metodologías de investigación, asignación y ponderación de las dimensiones de los estudios, y por la falta de unificación de los stakeholders sobre los que recaen dichas valoraciones", (OROZCO Y FERRÉ, 2012, p.16).

El World's Most Admired Companies surge de la asociación entre la consultora internacional Hay Group y la revista de negocios Fortune. Su universo de análisis se configura a paritr de los listados Fortune 1000 (compañías con mayores ingresos de EE.UU.) y Global 500 (compañías con mayores ingresos del mundo), cuyas fuentes son altos directivos (de las compañías seleccionadas), directores externos y analistas del sector. Los parámetros principales para la evaluación de la reputación son: Calidad de la dirección, calidad de los productos y servicios, innovación, inversión a largo plazo, solvencia financiera, capacidad de atraer, desarrollar y retener a empleados con talento, responsabilidad social con la comunidad y/o el medio ambiente, gestión acertada de los activos de la empresa, eficiencia en la realización de negocios a nivel mundial.

El monitor Global RepTrak Pulse lo realiza la consultora Ruputation Institute y se publica en la revista Forbes con el nombre de The World's Most Reputable Companies. Para determinar su universo realiza una selección de empresas resultantes de las encuestas realizadas a consumidores y líderes empresariales. Sus fuentes son consumidores de más de 40 países y líderes empresariales de 25 países. Los parámetros que utiliza para la evaluación son: Oferta de productos y servicios, innovación, entorno de trabajo, integridad, ciudadanía, liderazgo y resultados financieros

Por su parte, Merco trata de valorar las distintas facetas que conforman la reputación de una empresa. Para ello recopila los datos procedentes de diferentes fuentes de información y métodos: una encuesta a directivos, una 
evaluación de expertos, una evaluación directa, el Merco Tracking y el Merco Personas. Las variables de evaluación tenidas en cuenta en el cuestionario de méritos de reputación en la evaluación directa de Merco en el año 2013 son:

a. Los resultados económico financieros, teniendo en cuenta: a.1. El beneficio contable, el patrimonio, las ventas, el valor de la acción y el valor total en Bolsa a.2. La rentabilidad: recursos propios, rentabilidad por dividendos y las ventas por número de empleados.

b. La calidad de la oferta comercial, teniendo en cuenta los siguientes factores: b.1. Valores del producto: Cuota de mercado e informaciones que refuercen el conocimiento de los productos o servicios (certificaciones de calidad, premios y reconocimientos de calidad y reconocimientos y valoraciones de entidades independientes de la calidad del producto y servicio). b.2. Valores de marca e informaciones que puedan orientar sobre el valor de marca (premios y puesto en monitores de marca). También, acciones de comunicación, midiendo la inversión en publicidad-medios en España, los canales y medios de comunicación utilizados y el número de clientes en España, distinguiendo entre consumidores finales, distribuidores/empresas y Administraciones Públicas. b.3. Servicio y atención al cliente. Teniendo en cuenta la encuesta de satisfacción de clientes y usuarios, concretamente los indicadores de satisfacción global, de la calidad de la atención al cliente, de calidad del producto/servicio, de fidelización y de recomendación. Informaciones o estadísticas sobre el nivel de satisfacción y/o fidelización de los clientes y acciones de protección de los usuarios como defensor del cliente, Junta de Arbitraje u otros procedimientos. b.4 Responsabilidad sobre los productos de la empresa (aspectos tales como la salud y la seguridad, el etiquetado de los productos o servicios, las comunicaciones comerciales, la protección de la privacidad del cliente y las reclamaciones y resoluciones. Cabe destacar, que en los aspectos de salud y seguridad y de etiquetado de los productos o servicios se solicitan los indicadores del GRI PR1 y PR3 respectivamente (estándar más utilizado como marco para elaborar informes de sostenibilidad).

c. Reputación interna: c.1. Indicadores de gestión de personas (hasta 25 indicadores de calidad laboral, las horas y la inversión en formación, empleados con discapacidad o flexibilidad laboral). c.2. Indicadores de clima sociolaboral (hasta 16 indicadores como valores éticos y profesionales de la empresa, desarrollo profesional y promoción interna y capacidad de la empresa para atraer talento. c.3. Empleo. Número de empleados, porcentaje 
de empleados fijos, tipos de contratos, contratación de colectivos específicos y otras acciones destacables en el plano del empleo. c.4. Reconocimientos en el ámbito laboral. Identificar la presencia en monitores o rankings e indicar si disponen de reconocimientos y menciones.

d. Ética y responsabilidad corporativa: d.1. Comportamiento corporativo ético. Códigos éticos asumidos, organismos de autorregulación y buen gobierno de la sociedad (número total de miembros del Consejo de Administración, de Consejeros con cargo ejecutivo, de Consejeros independientes y de mujeres en el Consejo, y se comprueba si se dispone de una Comisión de Responsabilidad Corporativa/Reputación o Ética en el Consejo de Administración, se comprueba también si se dispone de un Consejero independiente en Responsabilidad Corporativa, si se realiza alguna evaluación externa de su gobierno corporativo y si se publica la retribución de los consejeros). d.2. Compromiso con la comunidad. Política expresa o estrategia de acción social de la empresa que defina su contribución a la comunidad local. Forma de canalizar dicha acción. Mecanismos para evaluar el retorno de la inversión y actividades realizadas en materia de patrocinio no comercial o de mecenazgo, actividades realizadas en acciones sociales y ONG y fomento del voluntariado. d.3 Responsabilidad social y medioambiental. Premios obtenidos, reconocimientos en índices de Sostenibilidad y Monitores, certificaciones o normas de RSC como la SGE 21, la SA 8000, la ISO 26000 o la RS 10 y si se dispone de certificaciones de calidad medioambiental como la ISO 14001 y EMAS. También se comprueba que políticas activas de preservación del medio ambiente y de responsabilidad social se llevan a cabo. Se miden que directrices o códigos tienen sobre gestión medioambiental y de responsabilidad social (Principios Ecuador, Global Compact, Directrices OCDE, Libro Verde...), si utilizan las directrices GRI para la elaboración de sus memorias y qué calificación tienen, también se comprueba que estándares de verificación externa de la memoria de responsabilidad social tienen (Norma ISAE 3000 o Norma AA 1000 AS) y por último que acciones realizan de protección o recuperación medioambiental. d.4. Proveedores. Se identifica si existe una política expresa de compras y/o procedimientos y sistemas al respecto, se comprueba si se tienen en cuenta criterios de responsabilidad o de sostenibilidad de los proveedores a la hora de contratarlos y se especifica si se realiza algún tipo de auditoría. Además se comprueba que porcentaje hay de compra a proveedores locales donde se desarrollan las actividades de la empresa, concretamente se pide el indicador GRI EC6, también se com- 
prueba que porcentaje de proveedores, contratistas y otros socios que han sido objeto de análisis en materia de derechos humanos, concretamente se solicita el indicador GRI HR2, y por último se pide el numero de proveedores significativos en los que el derecho a la libertad de asociación y de acogerse a convenios colectivos pueda ser violentado, concretamente se solicita el indicador GRI HR5.

e. Dimensión internacional de la empresa: e.1. Expansión internacional. Número de países con presencia institucional estable (propia o a través de participación). e.2. Evolución de la presencia internacional institucional. Número de países en los que opera, número de empleados fuera de España, nivel de ventas, porcentaje de aportaciones de otros países al resultado global y número de clientes.

f. Innovación: f.1. Inversiones en I+D. Resultados de inversión directa y en centros de investigación y universidades. f.2. Nuevos productos y servicios. Número de patentes registradas, número de modelos de utilidad registrados, número de modelos industriales registrados y número de otros registros. f.3. Otros aspectos. Aspectos respecto a la innovación como premios, menciones y proyectos financiados o subvencionados por las Administraciones Públicas.

g. Gestión de la Reputación Corporativa: Área de gestión para la Reputación Corporativa, plantilla del departamento y su nivel de dependencia jerárquica. Existencia o no de un modelo o sistema de gestión de la Reputación Corporativa y reconocimientos y menciones en este ámbito.

\section{RESULTADOS}

El Apartado 1 del GRI, estrategia y análisis, no se recoge en el cuestionario de méritos de reputación del Merco. Esta información va enfocada a proporcionar una visión estratégica de la organización con respecto a la sostenibilidad que en el Merco no se recoge.

En el apartado 2 del GRI hay dos indicadores que coinciden con los de Merco: 1. Número y nombre de países en los que opera la organización recogido en el punto 5.1 del Merco, expansión internacional. 2. Premios y las distinciones distribuidos en diferentes puntos en el Merco (apartados 2.1, 2.2, 3.4, 4.3, 6.3 y 7). Mientas que la información solicitada por el GRI pretende establecer un contexto general para comprender el desempeño de la organización, desde el Merco se solicita un volumen mayor de información 
sobre la dimensión internacional de la organización y sobre los premios, distinciones y reconocimientos que se han obtenido.

En el apartado III "parámetros de la memoria" del GRI, no hay ningún punto de coincidencia, ya que se trata de descriptores específicos de la memoria de sostenibilidad.

En el apartado IV del GRI, gobierno, compromisos y participación de los grupos de interés, hay un aspecto en común, de quince posibles, con los indicadores de evaluación utilizados por Merco, se trata del número y sexo de los miembros del máximo órgano de gobierno. Mientras que el GRI solicita información sobre los grupos de interés para describirlos y determinar el nivel de participación en la elaboración de la memoria, los indicadores Merco van más enfocados a determinar el nivel de satisfacción del cliente.

El apartado V del GRI, enfoque de gestión e indicadores de desempeño y descripción, está dividido a su vez en tres subapartados: dimensión económica (V.1), dimensión ambiental ( $\mathrm{V} .2$ y dimensión social (V.3). En cuanto a la dimensión económica (V.1) se observa que de los nueve indicadores que lo definen, tan solo dos coinciden con los evaluados desde el Merco, el EC1 valor económico directo y el EC6 gastos en proveedores locales (políticas, prácticas y proporción). Este último se solicita en el cuestionario de Merco directamente como indicador EC6 del GRI. En cuanto a la dimensión económica del Merco los indicadores van más enfocados a los resultados financieros como el beneficio y la rentabilidad, mientras que el GRI la dimensión económica va enfocada a la sostenibilidad y su impacto en la organización.

En el subapartado dimensión ambiental (V.2) no coincide ningún indicador ya que está enfocada a los impactos de una organización en los sistemas naturales vivos e inertes, incluidos los ecosistemas, el suelo, el aire y el agua. Aunque hay que destacar que en la evaluación directa del Merco, en el apartado 4.2 responsabilidad social y medioambiental se pide que se indiquen las certificaciones o normas de RSC y concretamente se solicitan las directrices GRI que utilizan para la elaboración de sus memorias y qué calificación tienen.

El subapartado dimensión social (V.3) se divide a su vez en cuatro tipos de indicadores. Los indicadores del desempeño de prácticas laborales y ética del trabajo (V.3.1) coinciden en 6 aspectos con el cuestionario de Merco, concretamente en el apartado de reputación interna con los indicadores de gestión de personas y los indicadores de clima sociolaboral. En cuanto a los indicadores del desempeño de los Derechos Humanos (V.3.2) desde el apartado de proveedores del cuestionario del Merco se solicitan directamente 
dos indicadores GRI. Los indicadores de desempeño de Derechos Humanos exigen que las organizaciones informen como se han implantado procesos para el cumplimiento en plantilla y proveedores. En el cuestionario del Merco se solicitan indicadores similares a nivel de proveedores pero a nivel de trabajadores no existe ningún indicador que compruebe el cumplimiento de dichos derechos.

Con respecto a los indicadores del desempeño de Sociedad (V.3.3) se observa que no hay ninguna coincidencia. Los indicadores de desempeño social del GRI centran su atención en los impactos que las organizaciones tienen en las comunidades locales en las que operan y aclaran cómo se gestionan los riesgos que pueden aparecer a partir de sus interacciones con otras instituciones sociales. En el Merco dentro del apartado ética y responsabilidad corporativa encontramos indicadores que miden el compromiso con la comunidad pero no reflejan que impactos concretos, sino que se comprueba qué políticas o estrategias de acción social llevan a cabo, cómo se canalizan y qué mecanismos disponen para evaluar el retorno de la inversión.

Por último, los indicadores del desempeño de Responsabilidad de productos (V.3.4) coinciden plenamente en dos aspectos con el apartado de responsabilidad sobre los productos de la empresa del Merco, donde se solicitan directamente como indicadores $\mathrm{GRI}$, dichos aspectos se refieren a salud, seguridad y etiquetado de los productos o servicios.

En cuanto el cuestionario de evaluación directa del Merco se observa que dispone de indicadores que no se tienen en cuenta en la memoria de sostenibilidad GRI como los valores del producto y de la marca, la innovación (Inversiones en I+D y nuevos productos y servicios) y un apartado específico para comprobar la gestión de la Reputación Corporativa en la organización.

\section{CONCLUSIONES Y DISCUSIÓN}

Tras la exposición de los resultados, coincidimos con aquellos autores que afirman que existe una notable relación entre los conceptos Responsabilidad y Reputación (MARTÍN, FERNÁNDEZ y ALAMEDA, 2010). Esta relación se evidencia en dos consideraciones. En primer lugar, como se afirma en la introducción (VILLAFAÑE, 2009), la relación se produce en tanto la responsabilidad contribuye a la formación de la reputación corporativa de una institución. En segundo lugar, como se aprecian en los resultados, existen diversos puntos de coincidencia en los sistemas de medición de ambos conceptos. 
Estas afirmaciones muestran a su vez que, si bien la relación entre ambos es evidente, también lo es que ambos conceptos hacen referencia a realidades distintas, a pesar de que muchas organizaciones se empeñen en realizar una gestión de la responsabilidad en el marco de los beneficios mercantiles (FERNÁNDEZ, 2007). Ambos conceptos, en sus definiciones, expresan claramente que su gestión es independiente una de otra y que los programas de responsabilidad de una institución tienen que ver con el modo en el que la institución manifiesta y ejerce su compromiso con la sostenibilidad. Así, dichos programas no deben ser acciones únicamente encaminadas a gestionar la imagen y la reputación corporativa de una empresa o medir sus resultados en función de la valoración de los stakeholder hacia los valores de la marca. Los resultados del análisis realizado muestran cómo la responsabilidad contribuye a la formación de la reputación, al menos contribuye al sistema establecido para medir la reputación de una institución. Así, algunos indicadores utilizados para evaluar la responsabilidad son utilizados para valorar la reputación de una organización.

Finalmente, entendemos que la existencia de coincidencias, en algunos apartados, entre los indicadores de la guía GRI y los indicadores no deben ser utilizadas para distorsionar la intencionalidad de los programas de responsabilidad de las organizaciones. Consideramos que este trabajo permite establecer una sólida base teórica para trazar investigaciones ocupadas en comprobar el compromiso de las organizaciones con la transparencia y las intenciones de sus programas de sostenibilidad y la realización y publicación de sus memorias de sostenibilidad. Mediante la revisión de las estructuras y los contenidos de estas memorias, podemos comparar y comprobar si estos documentos son utilizados como reflejo del compromiso responsable de la organización y cómo apoyo a la gestión de la reputación o, simplemente, como herramientas ideadas y diseñadas para contribuir a la creación de la imagen y la reputación corporativas.

\section{BIBLIOGRAFÍA}

BERBEL, G., REYES, J.D. y GÓMEZ, M. La Responsabilidad Social en las organizaciones (RSO): Análisis y comparación entre guías y normas de gestión e información. Revista Innovar, 17 (29), 27-48. 2007.

BOWEN, H.R. Social responsibilities of the businessman. New York: Harper. 1953.

CARRERAS, E., ALLOZA, A. y CARRERAS, A. Reputación Corporativa. Madrid: LID Editorial. 2013 
CARRILLO, V., CASTILLO, A. y TATO, J.L. El “Valor” de lo Intangible. La Gestión de la Reputación Corporativa. El caso de la marca TELEFONICA. Observatorio (OBS*) Journal, 7, 239-254. 2008.

DOPAZO, M.P. Informes de Responsabilidad Social Corporativa (RSC): Fuentes de Información y Documentación. Revista General de Información y Documentación, 22, 279305. 2012.

FERNÁNDEZ, J.M.R. Responsabilidad social corporativa y análisis económico: práctica frente a teoría. Ekonomiaz: revista vasca de economía, 65, 12-49. 2007.

FOMBRUM, C. y VAN RIEL, C. The Reputational Landscape. Corporate Reputation Review 1, 5-13. 1997.

ESTUDIO CORRESPONSABLE, Los informes de responsabilidad: presente y futuro. 2013. Recuperado el 20 de marzo de 2014, de http://www.corresponsables.com/webfm_ send/81

MARTíN, I., FERNÁNDEZ, E., y ALAMEDA, D. La RSC como estrategia de comunicación para lograr el incremento de la reputación corporativa. Actas del II Congreso Asociación Española de Investigación de la Comunicación. Málaga. Recuperado el 29 de enero de 2014, de http://www.aeic2010malaga.org/upload/ok/90.pdf. 2010.

MARTÍNEZ, I.M. y OLMEDO, I. La medición de la reputación empresarial: problemática y propuesta. Investigaciones Europeas 15, 127 - 142. 2009.

MONEVA, J.M. Información sobre responsabilidad social corporativa: Situación y tendencias. Revista Asturiana de economía, 34, 43-67. 2005.

MONITOR EMPRESARIAL DE REPUTACIÓN CORPORATIVA, MERCO. El proceso de elaboración del Merco España. 2013. Recuperado el 10 de abril de 2014, de http://www.merco. info/datafiles/0000/4004/El_proceso_de_elaboracion_de_Merco_2013.pdf.

OROZCO, J. y FERRÉ, C. Los índices de reputación corporativa y su aplicación en las empresas de comunicación. Actas del III Congreso Internacional Asociación Española de Investigación de la Comunicación. Tarragona. Recuperado el 10 de diciembre de 2013, de http://www.ae-ic.org/tarragona2012/contents/comunicacions_cd/ok/95.pdf. 2012.

VILLAFAÑE, J. La buena reputación: Claves del valor intangible de las empresas. Madrid: Ediciones Pirámide. 2004.

VILLAFAÑE, J. La comunicación empresarial y la gestión de los intangibles en España y Latinoamérica. Madrid: Pirámide. 2005.

VILLAFAÑE, J. Reputación corporativa y RSC. Bases empíricas para un análisis. Telos:

Revista de pensamiento sobre tecnología y sociedad, 79, 75-81. 2009. 


\section{Miguel Ángel Nicolás Ojeda}

Doctor en Comunicación e investigador principal del grupo "Publicidad y Relaciones Públicas, redefiniciones, públicos y medios" de la UCAM. Actualmente es profesor de las asignaturas Planificación de Medios, Leguaje Publicitario y Taller de Producción Audiovisual Publicitaria en el Grado de Publicidad y Relaciones Públicas de la UCAM y en el Master Oficial en Dirección de Comunicación.

\section{David Sánchez Hervás}

Doctorando y ayudante de investigación del grupo "Publicidad y Relaciones Públicas, redefiniciones, públicos y medios" de la UCAM. Licenciado en Periodismo, con itinerario de especialización en comunicación institucional y de empresas, y con Máster Oficial en Dirección de Comunicación. 
